\title{
Percepçáo da competência ocupacional e comportamentos sexuais de risco em toxicodependentes ${ }^{1}$
}

\author{
Celso Xavier de Melo Teixeira
}

Instituto Politécnico do Porto, Escola Superior de Tecnologia da Saúde do Porto, Porto, Portugual

\begin{abstract}
Resumo: Objetivo: Estudar/compreender os comportamentos sexuais de risco e a percepção da competência ocupacional em toxicodependentes, em função da presença/ausência de Doenças Sexualmente Transmissíveis (DST). Método: Optou-se por uma metodologia quantitativa e transversal, assumindo o estudo um carácter exploratório e descritivo. Assim, 166 indivíduos toxicodependentes, com 34,6 anos de média de idade, foram avaliados com os seguintes instrumentos: Auto-Avaliação Ocupacional e QC-VIH. Resultados: Nos comportamentos sexuais, os toxicodependentes infetados por HIV eram o grupo com a maior frequência de utilização do preservativo e um maior conhecimento em relação ao HIV. Por outro lado, os toxicodependentes sem DST apresentaram valores medianos mais altos para a percepção da competência ocupacional. Conclusões: São diferentes os comportamentos sexuais entre toxicodependentes, de acordo com a sua serologia. Os toxicodependentes sem DST continuam a manter comportamentos sexuais de risco. Parecem não ter a percepção da gravidade para a sua saúde e para a competência ocupacional do poder vir a ser infetado pelo HIV. Quando um toxicodependente é confrontado com a notícia da sua seropositividade ao HIV parece existir um comportamento comum quanto ao uso de precauções (preservativo), redução dos riscos em todo o tipo de relações sexuais e diminuição da percepção da competência ocupacional.
\end{abstract}

Palavras-chave: Dependência de Heroína, Transtornos Relacionados ao Uso de Cocaína, Doenças Sexualmente Transmissiveis, Fatores Sexuais, Terapia Ocupacional.

\section{Perception of occupational competence and sexual risk behaviors in drug addicts $^{2}$}

\begin{abstract}
Objective: This study aimed to study/understand sexual risk behaviors and the perception of occupational competence in drug addicts, regarding the existence/absence of Sexually Transmitted Diseases (STD). Method: We opted for a quantitative and transversal methodology that provided an exploratory and descriptive nature to the study. To this end, 166 drug addicts aged 34.6 years on average were assessed through the following questionnaires: “Occupational Self Assessment" and "HIV-KQ". Results: Regarding sexual behaviors, drug addicts infected with HIV were the group using condoms more frequently and having a more comprehensive knowledge about HIV. On the other hand, drug addicts with no STDs presented higher median scores related to occupational competence, volition, and performance ability when compared with the other two groups. Conclusions: Sexual risk behaviors among drug addicts differ according to their serology. Drug addicts without STD maintain sexual risk behaviors. They seem to have no perception of how serious the fact of being infected with HIV is to their health and occupational ability. When a drug addict is confronted with his own seropositivity to HIV, there seems to be a common behavior related to the use of precautions (condoms), risk reduction in every type of sexual relation, and decreased perception of occupational competence.
\end{abstract}

Keywords: Heroin Dependence, Cocaine-related Disorders, Sexually Transmitted Diseases, Sex Factors, Occupational Therapy.

Autor para correspondência: Celso Xavier de Melo Teixeira, Instituto Politécnico do Porto, Escola Superior de Tecnologia da Saúde do Porto, Rua Valente Perfeito, 322, 4400-330 Vila Nova de Gaia, Porto, Portugal, e-mail: celso.teixeira@idt.min-saude.pt; cxt@estsp.ipp.pt Recebido em 29/8/2013; Revisão em 18/2/2014; Aceito em 4/5/2014. 


\section{Introdução}

Competência ocupacional é o grau em que cada indivíduo mantém o padrão de participação ocupacional satisfatório e produtivo em papéis das diferentes áreas de ocupação, tendo em conta os seus valores e padróes de desempenho (KIELHOFNER, 2002).

Quando a um toxicodependente passa a estar associada também uma DST, vai-se acentuando o desleixo, a falta de cuidados em relaçáo à saúde e bem-estar e os déficits no desempenho ocupacional das diferentes áreas de ocupaçáo (ANANDAN et al., 2006). Quando um toxicodependente infectado por HIV utiliza as diferentes estruturas de tratamento, o papel sexual passa a ser o principal, em relação aos outros papéis ocupacionais e aspectos do tratamento (ZHAO et al., 2006). Com efeito, é a infeção por HIV na população toxicodependente aquela que traz mais consequências para o equilíbrio ocupacional e para a qualidade de vida dos indivíduos (KALICHMAN, 1998). Aqueles toxicodependentes que não estão infectados com alguma DST têm uma melhor autoavaliação da sua competência ocupacional, pois possuem crítica em relação ao que são, ou não, capazes de fazer em outras áreas de ocupação (BRAVEMAN; KIELHOFNER, 2006).

Os consumidores de drogas são um dos principais grupos de risco, de transmitir ou contrair a infeção por HIV, devido a diferentes comportamentos (NEGREIROS; MAGALHÃES, 2005; CORVINELLI, 2007). O acréscimo simultâneo de consumo de drogas e de comportamentos sexuais de risco corresponde a uma realidade empírica (ROSENGARD; ANDERSON; STEIN, 2006; ZHAO et al., 2006). Consideram-se comportamentos sexuais de risco os comportamentos que aumentam a probabilidade de morbidez (i.e., doença e danos) e mortalidade (ADLER; KEGELES; GENEVRO, 1992).

Por esses motivos, surgiu este trabalho de investigação, o qual pretende aferir a percepção da competência ocupacional e os comportamentos sexuais de risco dos consumidores de drogas, de acordo com o seu estado serológico em relação ao HIV. Apesar da relevância do tema, a informação relativa a uma eventual afetação no desempenho ocupacional dos toxicodependentes é escassa na literatura científica. Desconhecemos a existência de estudos que dediquem atenção preferencial à avaliação da competência ocupacional em toxicodependentes seropositivos para HIV. Na investigação de metanálise desenvolvida por Stoffel e Moyers (2004), verifica-se, de acordo com as diferentes valências profissionais que intervêm com a população toxicodependente, uma carência de estudos que examinem a eficácia e efetividade da intervenção ocupacional com essa população específica. Quanto aos estudos dos comportamentos de risco nos toxicodependentes, esses incidem majoritariamente na compreensão dos comportamentos que são inerentes à partilha de material de uso endovenoso e menos nos comportamentos sexuais de risco (LOXLEY, 2000; AZEVEDO; BOTEGA; GUIMARÃES, 2007).

O problema central desta investigação é: Quais os comportamentos dessa população associados à transmissão do HIV por via sexual? Procuramos também perceber se esses comportamentos sexuais estáo de acordo com os comportamentos de risco associados à transmissão por via sanguínea. E de que forma a seropositividade tem interferência, de forma global, na perceção da competência ocupacional dos indivíduos e, de forma específica, na motivação para se envolver em novas ocupaçóes, na capacidade de utilizar as aptidôes relacionadas com a comunicaçâo e no desempenho eficiente dos diferentes papéis ocupacionais.

Muitas vezes, a avaliação ocupacional percebida pelo indivíduo é desvalorizada em relaçâo às suas reais capacidades. Salz (2001) diz-nos que a mudança do paradigma, em relação à infeção por HIV, de uma doença mortal para uma doença crônica e dispendiosa aumenta e altera as expectativas de vida. Ou seja, faz com que o papel profissional seja considerado fundamental para a melhoria da qualidade de vida das pessoas infectadas por HIV. A despreocupação e desleixo para consigo e para com a saúde entre a populaçáo toxicodependente vai acentuar negativamente a sua avaliação da competência ocupacional (BRAVEMAN; KIELHOFNER, 2006). O envolvimento satisfatório no papel profissional faz com que os indivíduos infectados por HIV realizem uma autoavaliação positiva das competências de desempenho dos outros papéis ocupacionais (KIELHOFNER et al., 2004) e é um fator protecionista em relação à adoção de comportamentos de risco de infecção pelo HIV (NEGREIROS, 2006).

A sexualidade tem muita importância no desenvolvimento dos indivíduos e é essencial para a vida das pessoas como o são todas as ocupaçôes em que elas se envolvem, desde cuidar de si próprias, apreciar a vida ou contribuir para a construçáo social e econômica de sua comunidade (JONES; WEERAKOON; PYNOR, 2005). Mas não é consensual na literatura considerar o papel sexual como um papel ocupacional. Couldrick (2005) concorda com a inclusão do papel sexual como um 
papel ocupacional, como parte de uma abordagem holística e humanística de todos os cuidados de saúde.

O papel sexual proporciona uma organização longitudinal do tempo e espaço do indivíduo. $\mathrm{O}$ modelo teórico que se centra no pressuposto de que os diferentes papéis ocupacionais encerram uma extrema importância para a organização temporal da vida do indivíduo é o Modelo de Ocupação Humana (MOH) (KIELHOFNER, 2002).

$\mathrm{O} \mathrm{MOH}$ enfatiza que viver com uma incapacidade, seja de origem física e/ou psicológica (como a toxicodependência ou infeção por HIV), requer mais do que minimizar as limitaçôes funcionais; requer que os indivíduos estabeleçam uma rotina de vida e relações sociais a vários níveis. Numa situação específica como é a infeção por HIV, o cliente deve ser ativo na adequação face às circunstâncias, sendo para esse efeito relevante: colaborar na definição dos seus déficits, na definição dos seus aspectos positivos e no estabelecimento de objetivos terapêuticos - percepção da competência ocupacional (FISH; RUDMAN, 1998). De acordo com o MOH, para além do ambiente físico e social, são três os fatores principais que influenciam a competência ocupacional, os quais também servem hierarquicamente para descrever a estrutura da ocupação (BARON et al., 2003; SOUSA, 2006): Volição, Habituação e Capacidade de Desempenho.

\section{Método}

\subsection{Enquadramento e objetivos}

De acordo com as questôes levantadas na revisão bibliográfica, destacam-se três aspetos teóricos centrais da literatura: os toxicodependentes têm mais cuidados preventivos na transmissáo do HIV por via endovenosa do que por via sexual (e.g., Deren et al. (2008) e Lam (2008)); os toxicodependentes, ao serem portadores do HIV, diminuem os comportamentos sexuais de risco (e.g., Guerra (1998) e Negreiros (2006)); e a perceção global da competência ocupacional diminui após a infecção por HIV (e.g., Kielhofner et al. (2004) e Braveman e Kielhofner (2006)). Essa informação sugere que na população toxicodependente o equilíbrio e a satisfação entre os diferentes papéis ocupacionais, bem como os comportamentos sexuais, podem se diferenciar em função do estado serológico dos indivíduos.

No seguimento dessa linha de pensamento, o principal objetivo deste trabalho é: estudar e compreender os comportamentos sexuais de risco de infecção e a percepção da competência ocupacional numa amostra de toxicodependentes da Zona Norte de Portugal em função da presença/ausência de DST e HIV.

Definimos como hipótese da investigação a seguinte: A população toxicodependente infectada por HIV apresenta menos comportamentos sexuais de risco e índices inferiores de competência ocupacional em relação à população toxicodependente não infectada por HIV.

Optamos por recorrer a uma metodologia quantitativa e transversal, tendo como universo de estudo os indivíduos toxicodependentes da Zona Norte de Portugal. O presente estudo apresenta carater exploratório e descritivo (FORTIN, 2003).

\subsection{Amostra}

A amostra foi obtida através do método não probabilístico de amostragem por conveniência (FORTIN, 2003) no local de desenvolvimento do estudo: Unidade de Desabituação do Norte (UD-N), a partir da população de indivíduos acompanhados em regime de internamento.

A seleção da amostra decorreu no período de maio a outubro (seis meses), sendo constituída por todos os indivíduos que cumpriam os critérios predefinidos (e.g., critério de inclusão - apresentarem uma história de pelo menos dois anos sem interrupçôes com consumo regular de heroína e/ou cocaína; critério de exclusão - ter o álcool como principal substância psicoativa de consumo) e que aceitaram participar do estudo.

\subsection{Instrumentos}

Com vistas à obtenção dos dados necessários para a realização deste estudo, construiu-se e utilizou-se um questionário sociodemográfico introdutório, com o qual também se obtiveram as informaçóes sobre o comportamento sexual. Os outros dois instrumentos utilizados foram:

Autoavaliaçáo ocupacional (OSA), versão traduzida, adaptada e validada para a populaçáo portuguesa por Sousa (2006) do The Occupational Self Assessment desenvolvido por Baron et al. (2003). É um instrumento de avaliação (de autopreenchimento), baseado no $\mathrm{MOH}$, indicado para a população adulta, desenvolvido para avaliar a percepção que cada indivíduo tem de sua competência ocupacional.

Questionário de conhecimento de VIH (QC-VIH), na versão traduzida e adaptada para a língua portuguesa por Cruz (1998) do HIV 
Knowledge Questionnaire desenvolvido por Carey, Morrison-Beedy e Johnson (1997). Trata-se de um instrumento de avaliação desenvolvido para avaliar o grau de conhecimento em relação à transmissão, prevenção e consequências da infecção pelo HIV.

\subsection{Procedimentos}

A informaçáo obtida foi analisada de forma estatística, segundo uma metodologia quantitativa, com recurso ao programa informático Statistical Package for Social Science - SPSS (versão 16.0 para Microsoft Windows) (PEREIRA, 2004).

Depois da análise das características da amostra e face aos resultados encontrados, optou-se por dividir os indivíduos de acordo com a presença/ausência de DST, de forma a verificar como essa variável influencia a percepção da competência ocupacional e os comportamentos sexuais. Dividiu-se a amostra em 3 grupos:

- Grupo A: Toxicodependentes sem qualquer DST;

- Grupo B: Toxicodependentes portadores de outras DST que não o HIV;

- Grupo C: Toxicodependentes portadores do HIV.

Para facilidade de análise dos resultados optou-se por somar as pontuaçóes dadas na escala de Likert do instrumento OSA, agrupando as questóes pelas dimensôes: competência ocupacional, valores e satisfação ocupacional, e pelas sub-escalas: volição, habituação e capacidade de desempenho - descritas pelos autores.
Para testar a normalidade das variáveis valemo-nos do teste Kolmogorov-Smirnov (KS). Para comparar os resultados médios obtidos nos três grupos definidos, recorreu-se a testes paramétricos e não paramétricos, nomeadamente a ANOVA (F) ou o teste Kruskall-Wallis $(\mathrm{H})$, conforme se tratasse de variáveis normais ou não normais. Recorreu-se, igualmente, à MANOVA para a comparação dos valores das dimensóes e sub-escalas da OSA nos grupos, de acordo com os diferentes comportamentos sexuais. Para comparar resultados de proporçôes, utilizou-se o teste do Qui-Quadrado $\left(\chi^{2}\right)$. O nível máximo de significância escolhido foi de $5 \%$.

\section{Resultados}

A amostra constituiu-se de 166 indivíduos que acederam voluntariamente a colaborar no estudo, sendo em maior número (137-82,5\%) do gênero masculino (Tabela 1).

Em relação às DST, dividiu-se a amostra em três grupos: $46,4 \%$ dos indivíduos não indicam nenhuma DST (grupo A); 33,7\% têm alguma DST mas não são portadores do HIV (grupo B); e 19,9\% são portadores do HIV (grupo C).

Relativamente à Substância Habitual de Consumo (cf. Tabela 2), foi encontrada uma diferença significativa entre os grupos $\left(\chi^{2}=11,63 ; p=0,020\right)$, o que se traduz pelo fato de a maior parte dos toxicodependentes infectados por HIV $(63,6 \%)$ elegerem as duas substâncias em simultâneo como habituais de seu consumo. E é também esse grupo $\mathrm{C}$ o que apresenta maior percentagem de Consumo de Outras Substâncias Secundárias.

Tabela 1. Caraterísticas sociodemográficas da amostra.

\begin{tabular}{|c|c|c|c|}
\hline & & \multicolumn{2}{|c|}{$\mathrm{N}=166$} \\
\hline \multirow[t]{4}{*}{ Idade } & Média (anos) & \multicolumn{2}{|c|}{34,6} \\
\hline & DP & \multicolumn{2}{|c|}{8,1} \\
\hline & Mínimo - Máximo & \multicolumn{2}{|c|}{$18-57$} \\
\hline & & $\mathbf{N}$ & $\%$ \\
\hline \multirow[t]{2}{*}{ Gênero } & Masculino & 137 & 82,5 \\
\hline & Feminino & 29 & 17,5 \\
\hline \multirow[t]{3}{*}{ Estado civil } & Solteiro & 84 & 50,6 \\
\hline & Casado/União de fato & 43 & 25,9 \\
\hline & Divorciado/Separado/Viúvo & 39 & 23,5 \\
\hline \multirow[t]{5}{*}{ Situação laboral } & Empregado & 32 & 19,3 \\
\hline & Desempregado & 108 & 65,1 \\
\hline & De baixa médica & 12 & 7,2 \\
\hline & Estudante & 7 & 4,2 \\
\hline & Outra & 7 & 4,2 \\
\hline
\end{tabular}


No que diz respeito às variáveis sociodemográficas por grupo são de salientar alguns resultados (cf. Tabela 3), principalmente no que concerne à média de idade, meio de residência e tipo de habitação.

$\mathrm{Na}$ Tabela 4 encontra-se a análise das diferenças entre grupos para a experiência profissional, situação laboral atual e fonte de rendimento principal.

Em relação às demais questôes familiares por grupo (com quem vive, como considera o ambiente na sua família, se tem ou não filhos e se tem familiares que consomem drogas), não foi encontrado nenhum resultado estatisticamente significativo. O mesmo acontece em relação a parceiro sexual atual, idade da primeira relação sexual e duração da relação atual.

Nos comportamentos sexuais por grupo verificam-se diferenças estatisticamente significativas em duas variáveis: frequência de utilização do preservativo $\left(\chi^{2}=32,13 ; \mathrm{p}=0,000\right)$, o grupo $\mathrm{C}$ apresenta uma maior percentagem de Sempre usar preservativo $(75,8 \%)$; e em relação à frase Os preservativos reduzem o prazer das relações sexuais $\left(\chi^{2}=17,22 ; \mathrm{p}=0,009\right)$, a maior percentagem de Discordo totalmente é do grupo C $(45,5 \%)$ e a maior percentagem de Concordo totalmente, do grupo A (27,2\%), enquanto no grupo B as respostas são mais equitativas.

Nos outros comportamentos sexuais registram-se semelhanças entre os três grupos: mais de metade de cada grupo teve/tem encontros sexuais casuais com desconhecidos; mais de metade de cada grupo refere recusar sempre ter relaçóes sexuais com parceiro(a) casual por falta de preservativo; a maior percentagem em todos os grupos é para um parceiro sexual no

Tabela 2. Substâncias consumidas por grupo.

\begin{tabular}{|c|c|c|c|c|c|c|}
\hline & & $\begin{array}{c}\% \text { grupo A } \\
(\mathrm{n}=77)\end{array}$ & $\begin{array}{c}\% \text { grupo B } \\
(\mathrm{n}=56)\end{array}$ & $\begin{array}{c}\% \text { grupo C } \\
(\mathrm{n}=33)\end{array}$ & & \\
\hline & & Sem IST & $\begin{array}{l}\text { Com IST } \\
\text { sem VIH }\end{array}$ & Com VIH & $\chi^{2}$ & $\mathrm{p}$ \\
\hline \multirow{3}{*}{$\begin{array}{l}\text { Substância habitual } \\
\text { de consumo }\end{array}$} & Heroína & 32,5 & 28,6 & 12,1 & \multirow{3}{*}{$11,63^{*}$} & \multirow{3}{*}{0,020} \\
\hline & Cocaína & 6,5 & 21,4 & 24,2 & & \\
\hline & Heroína + cocaína & 61 & 50 & 63,6 & & \\
\hline \multirow{2}{*}{$\begin{array}{l}\text { Consumo de } \\
\text { outras substâncias } \\
\text { secundárias }\end{array}$} & Sim & 37,7 & 62,5 & 81,8 & \multirow{2}{*}{$20,20 * *$} & \multirow{2}{*}{0,000} \\
\hline & Não & 62,3 & 37,5 & 18,2 & & \\
\hline
\end{tabular}

$* \mathbf{p}<0,05$. ** $\mathbf{p}<0,01$.

Tabela 3. Variáveis sociodemográficas por grupo.

\begin{tabular}{|c|c|c|c|c|c|c|c|c|c|c|c|}
\hline \multirow[t]{2}{*}{ Grupos } & \multicolumn{3}{|c|}{ Grupo A } & \multicolumn{3}{|c|}{ Grupo B } & \multicolumn{3}{|c|}{ Grupo C } & \multirow[b]{2}{*}{$\mathbf{F}$} & \multirow[b]{2}{*}{$\mathrm{p}$} \\
\hline & $\mathbf{M}$ & DP & $\mathrm{n}$ & $\mathbf{M}$ & DP & $\mathrm{n}$ & $\mathbf{M}$ & DP & $\mathrm{n}$ & & \\
\hline Idade & 33 & 8,3 & 77 & 37 & 8,2 & 56 & 36 & 6,9 & 33 & $4,24 *$ & 0,016 \\
\hline & & $\%$ & & & $\%$ & & & $\%$ & & $\chi^{2}$ & $\mathrm{p}$ \\
\hline Gênero & & & & & & & & & & 3,28 & 0,194 \\
\hline Masculino & & 87 & & & 82,1 & & & 72,7 & & & \\
\hline Feminino & & 13 & & & 17,9 & & & 27,3 & & & \\
\hline Estado civil & & & & & & & & & & 4,46 & 0,347 \\
\hline Solteiro & & 53,2 & & & 46,4 & & & 51,6 & & & \\
\hline Casado/União de fato & & 29,9 & & & 21,4 & & & 24,2 & & & \\
\hline Divorciado/Separado/Viúvo & & 16,9 & & & 32,1 & & & 24,2 & & & \\
\hline Meio de residência & & & & & & & & & & $12,01 * *$ & 0,002 \\
\hline Meio rural & & 42,9 & & & 32,1 & & & 9,1 & & & \\
\hline Meio urbano & & 57,1 & & & 67,9 & & & 90,9 & & & \\
\hline Tipo de habitação & & & & & & & & & & $20,56 * *$ & 0,008 \\
\hline Apartamento & & 29,9 & & & 37,5 & & & 24,2 & & & \\
\hline Moradia & & 46,8 & & & 33,9 & & & 24,2 & & & \\
\hline Habitação degradada & & 14,3 & & & 12,5 & & & 27,4 & & & \\
\hline Quarto alugado/Pensão & & 6,5 & & & 7,1 & & & 24,2 & & & \\
\hline Outro & & 2,6 & & & 8,9 & & & 0,0 & & & \\
\hline
\end{tabular}

$* \mathbf{p}<0,05 . * * \mathbf{p}<0,01$. 
último mês; e há distribuição equitativa na resposta à questáo de se tinha alterado/mudado comportamentos e práticas sexuais desde que ouviram falar da SIDA.

Quanto aos comportamentos no último encontro/ relação sexual (cf. tabela 5), verificam-se diferenças estatisticamente significativas entre os grupos, no uso do preservativo e no pedido expresso do uso do preservativo.

Das respostas obtidas no QC-VIH - instrumento onde se avalia o grau de conhecimentos em relação à transmissão, prevenção e consequências da infecção pelo HIV - recorreu-se ao cálculo do alpha de Cronbach para determinar a consistência interna das respostas e o seu valor foi de 0,791 .

Verificou-se que a média de percentagem de respostas corretas é sempre superior no grupo C. O grupo A é aquele que apresenta a média de percentagem de respostas corretas mais baixa. Assim, e em relação ao total de respostas corretas do QC-VIH (variável normal pelo KS), verifica-se que a percentagem média de respostas corretas é de $60,8 \%$ para o grupo A, $65,9 \%$ para o grupo B e $71,2 \%$ para o grupo $C(F=3,48 ; p=0,033)$. $E$ de entre os 18 itens que constituem o QC-VIH, foram encontrados resultados estatisticamente significativos em quatro.

De referir ainda que para o total do QC-VIH, o mínimo de respostas corretas foram $4(22,2 \%)$ e o máximo, 17 (94,4\%).

No que se refere à OSA - desenvolvida para avaliar a percepção que o indivíduo tem da sua competência ocupacional, valores e satisfação ocupacional -, recorreu-se ao cálculo do alpha de Cronbach para determinar a consistência interna das respostas às três dimensôes. Os valores alpha de Cronbach da OSA foram 0,835 para a competência ocupacional, 0,902 para os valores e 0,874 para a satisfação ocupacional. De referir que esses resultados náo aumentam significativamente com a eliminação de quaisquer das sub-escalas, ou se algum dos itens que o compóem fosse eliminado.

A fim de verificar a existência de diferenças entre os grupos de indivíduos ao nível das dimensōes (competência ocupacional, valores e satisfação ocupacional) e sub-escalas (volição, habituação e capacidade de desempenho) analisadas através do

Tabela 4. Experiência profissional, situação laboral e fonte de rendimento principal, por grupo.

\begin{tabular}{|c|c|c|c|c|c|c|c|c|c|c|c|c|}
\hline & & \multicolumn{3}{|c|}{ Grupo A } & \multicolumn{3}{|c|}{ Grupo B } & \multicolumn{3}{|c|}{ Grupo C } & \multirow[b]{2}{*}{$\mathbf{H}$} & \multirow[b]{2}{*}{$\mathrm{p}$} \\
\hline & & M & DP & $\mathrm{n}$ & $\mathbf{M}$ & DP & $\mathrm{n}$ & $\mathbf{M}$ & DP & $\mathrm{n}$ & & \\
\hline & $\begin{array}{l}\text { Experiência } \\
\text { profissional (anos) }\end{array}$ & 9,4 & 7,1 & 77 & 9,2 & 8,1 & 56 & 7,5 & 6,6 & 33 & 2,07 & 0,356 \\
\hline & & & $\%$ & & & $\%$ & & & $\%$ & & $\chi^{2}$ & $\mathrm{p}$ \\
\hline \multirow{5}{*}{$\begin{array}{l}\text { Situação } \\
\text { laboral }\end{array}$} & Empregado & & 24,7 & & & 19,6 & & & 6,1 & & & \\
\hline & Desempregado & & 61,0 & & & 69,6 & & & 66,7 & & & \\
\hline & De baixa & & 9,1 & & & 7,1 & & & 3,0 & & $25,24 * *$ & 0,001 \\
\hline & Estudante & & 3,9 & & & 3,6 & & & 6,1 & & & \\
\hline & Outra & & 1,3 & & & 0,0 & & & 18,2 & & & \\
\hline \multirow{3}{*}{$\begin{array}{l}\text { Fonte de } \\
\text { rendimento } \\
\text { principal }\end{array}$} & Vencimento & & 31,2 & & & 23,2 & & & 12,1 & & & \\
\hline & $\begin{array}{l}\text { Subsídios/ } \\
\text { Rendimento mínimo }\end{array}$ & & 23,4 & & & 23,2 & & & 57,6 & & $16,27 * *$ & $\mathbf{0 , 0 0 3}$ \\
\hline & Outras & & 45,4 & & & 53,6 & & & 30,3 & & & \\
\hline
\end{tabular}

** $\mathbf{p}<\mathbf{0 , 0 1}$.

Tabela 5. Comportamentos no último encontro/relação sexual, por grupo.

\begin{tabular}{|c|c|c|c|c|c|c|}
\hline & & $\%$ grupo A & $\%$ grupo B & \% grupo C & $\chi^{2}$ & $\mathrm{p}$ \\
\hline \multirow{2}{*}{ Usou preservativo } & Não & 55,8 & 50,0 & 15,2 & \multirow{2}{*}{$16,02 * *$} & \multirow{2}{*}{0,000} \\
\hline & Sim & 44,2 & 50,0 & 84,8 & & \\
\hline \multirow{2}{*}{$\begin{array}{l}\text { Foi uma relação com o parceiro } \\
\text { habitual }\end{array}$} & Não & 20,8 & 35,7 & 24,2 & \multirow{2}{*}{3,82} & \multirow{2}{*}{0,148} \\
\hline & Sim & 79,2 & 64,3 & 75,8 & & \\
\hline \multirow{2}{*}{$\begin{array}{l}\text { Foi pedido expressamente o uso } \\
\text { do preservativo }\end{array}$} & Não & 58,4 & 58,9 & 24,2 & \multirow{2}{*}{$12,54 * *$} & \multirow{2}{*}{0,002} \\
\hline & Sim & 41,6 & 41,1 & 75,8 & & \\
\hline \multirow[t]{2}{*}{ Tinham preservativos disponíveis } & Não & 20,8 & 14,3 & 15,2 & \multirow{2}{*}{1,1} & \multirow{2}{*}{0,576} \\
\hline & Sim & 79,2 & 85,7 & 84,8 & & \\
\hline
\end{tabular}

** $\mathbf{p}<\mathbf{0 , 0 1}$. 
questionário OSA, foram realizadas análises de variância univariada (ANOVA). Como se vê na tabela 6 , todas as variáveis são normais pelo KS. Nas variáveis que apresentam valor da diferença entre grupos estatisticamente significativo verifica-se que os indivíduos do grupo A apresentam as médias mais elevadas na percepçáo dos diferentes domínios da OSA. O grupo C é o que apresenta os valores médios mais baixos, o que evidencia que os toxicodependentes infetados por HIV são os indivíduos que apresentam mais dificuldades no processo de experimentar, interpretar e escolher os comportamentos ocupacionais com vistas a um desempenho adequado e que esteja de acordo com o contexto em que se encontram.

Por último, verificou-se a existência de diferenças dos grupos entre a OSA, de acordo com os diferentes comportamentos sexuais, e conhecimento relativamente ao HIV, apresentados pelos indivíduos dos três grupos. Para tal foram conduzidas análises de variância multivariada (MANOVA). Na tabela 7 são apresentados os resultados estatisticamente significativos encontrados, de acordo com os diferentes comportamentos sexuais. $\mathrm{Na}$ Tabela 8 sáo apresentados os resultados encontrados, de acordo com o conhecimento relativamente ao HIV.

\section{Discussão}

Encontrar um emprego remunerado é uma das maiores dificuldades dos toxicodependentes, devido à dificuldade de cumprirem horários por, geralmente, estarem sem trabalho por longo período, ou por não apresentarem competências para o cargo. Essa dificuldade aumenta se estiver associada à infecção por HIV. Pelos dados verificamos que a percentagem mais baixa de empregados corresponde aos toxicodependentes infectados por HIV, seguidos dos toxicodependentes com DST sem HIV; a percentagem mais alta de empregados encontrada foi

Tabela 6. Diferenças nos resultados das dimensões da autoavaliação ocupacional (OSA), de acordo com os três grupos.

\begin{tabular}{lrrrrrrrrrrrrc}
\hline & \multicolumn{1}{c}{ Grupo A } & \multicolumn{1}{c}{ Grupo B } & \multicolumn{1}{c}{ Grupo C } & \multicolumn{1}{c}{ Total } \\
\cline { 2 - 15 } & M & DP & M & DP & M & DP & M & DP & Mínimo & Máximo & F & p \\
\hline $\begin{array}{l}\text { Competência } \\
\text { ocupacional }\end{array}$ & 60,0 & 6,7 & 57,4 & 7,8 & 53,3 & 7,3 & 57,8 & 7,6 & 35 & 78 & $\mathbf{9 , 6 6 * *}$ & $\mathbf{0 , 0 0 0}$ \\
Valores & 62,1 & 10,1 & 62,5 & 9,8 & 59,2 & 8,2 & 61,7 & 9,7 & 42 & 84 & 1,3 & 0,275 \\
$\begin{array}{l}\text { Satisfação } \\
\text { ocupacional }\end{array}$ & 2,0 & 10,4 & 5,1 & 12,7 & 6,0 & 11,4 & 3,8 & 11,5 & -19 & 41 & 1,91 & 0,151 \\
Volição & 14,6 & 2,5 & 13,9 & 2,5 & 12,2 & 2,2 & 13,9 & 2,6 & 7 & 19 & $\mathbf{1 8 , 4 6 * *}$ & $\mathbf{0 , 0 0 0}$ \\
Habituação & 13,3 & 2,5 & 12,8 & 2,9 & 12,1 & 2,1 & 12,9 & 2,6 & 6 & 19 & 2,63 & 0,057 \\
$\begin{array}{l}\text { Capacidade de } \\
\text { desempenho }\end{array}$ & 32,2 & 3,0 & 30,7 & 3,5 & 29,1 & 4,1 & 31,1 & 3,6 & 21 & 40 & $\mathbf{9 , 5 5 * *}$ & $\mathbf{0 , 0 0 0}$ \\
\hline
\end{tabular}

*** $\mathbf{p}<\mathbf{0 , 0 1}$.

Tabela 7. Diferenças nas dimensões e sub-escalas da OSA entre os grupos, em função dos comportamentos sexuais (MANOVA).

\begin{tabular}{|c|c|c|c|c|c|c|c|c|c|}
\hline & \multicolumn{8}{|c|}{ Comportamentos Sexuais } \\
\hline & & \multicolumn{2}{|c|}{$\begin{array}{c}\text { Frequência de } \\
\text { utilização do } \\
\text { preservativo }\end{array}$} & \multicolumn{2}{|c|}{$\begin{array}{c}\text { Número de } \\
\text { parceiros sexuais } \\
\text { no último mês }\end{array}$} & \multicolumn{2}{|c|}{$\begin{array}{l}\text { Frequência de } \\
\text { atividade sexual }\end{array}$} & \multicolumn{2}{|c|}{$\begin{array}{c}\text { Uso de preservativo } \\
\text { na última relação } \\
\text { sexual }\end{array}$} \\
\hline & & $\mathbf{F}$ & $\mathrm{p}$ & $\mathrm{F}$ & $\mathrm{p}$ & $\mathbf{F}$ & $\mathrm{p}$ & F & $\mathrm{p}$ \\
\hline \multirow{6}{*}{ 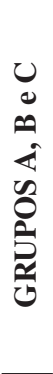 } & $\begin{array}{l}\text { Competência } \\
\text { ocupacional }\end{array}$ & 1,28 & 0,262 & $4,52^{*}$ & 0,035 & $8,56 * *$ & 0,004 & $15,85 * *$ & 0,000 \\
\hline & Valores & $5,75^{*}$ & $\mathbf{0 , 0 1 9}$ & 0,73 & 0,394 & 0,55 & 0,460 & $7,32 * *$ & 0,009 \\
\hline & $\begin{array}{l}\text { Satisfação } \\
\text { ocupacional }\end{array}$ & 1,29 & 0,260 & $4,31^{*}$ & 0,039 & $6,79 *$ & $\mathbf{0 , 0 1 0}$ & 0,01 & 0,982 \\
\hline & Voliçãao & $3,74^{*}$ & 0,047 & $4,77^{*}$ & $\mathbf{0 , 0 3 0}$ & 2,84 & 0,094 & 9,76 ** & 0,003 \\
\hline & Habituação & 1,26 & 0,265 & 3,13 & 0,079 & $7,65 * *$ & 0,006 & $12,12 * *$ & 0,001 \\
\hline & $\begin{array}{l}\text { Capacidade de } \\
\text { desempenho }\end{array}$ & 0,01 & 0,947 & 2,38 & 0,125 & $8,86 * *$ & 0,003 & $10,49 * *$ & 0,002 \\
\hline
\end{tabular}

$* \mathbf{p}<0,05$. ** $\mathbf{p}<0,01$. 
Tabela 8. Diferenças nas dimensões e subescalas da OSA entre os grupos, em função do conhecimento face ao VIH (MANOVA).

\begin{tabular}{|c|c|c|c|}
\hline & & \multicolumn{2}{|c|}{$\begin{array}{l}\text { Conhecimento em } \\
\text { relação ao VIH }\end{array}$} \\
\hline & & $\mathbf{F}$ & $\mathrm{p}$ \\
\hline \multirow{6}{*}{ 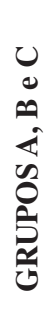 } & $\begin{array}{l}\text { Competência } \\
\text { ocupacional }\end{array}$ & 3,16 & 0,078 \\
\hline & Valores & $7,02 * *$ & 0,009 \\
\hline & Satisfação ocupacional & $11,71 * *$ & 0,001 \\
\hline & Voliçãa & 3,05 & 0,083 \\
\hline & Habituação & $5,89 *$ & 0,016 \\
\hline & $\begin{array}{l}\text { Capacidade de } \\
\text { desempenho }\end{array}$ & 0,44 & 0,510 \\
\hline
\end{tabular}

$* \mathbf{p}<0,05$. ** $\mathbf{p}<0,01$.

dos toxicodependentes sem DST, ou seja, esse fator é um dos indicadores do desajuste na adaptaçáo social. Kielhofner (2002) aponta a atividade profissional como um aspeto central na vida do adulto, uma vez que é onde a pessoa emprega grande parte do seu tempo, tornando-se uma importante componente da sua identidade ocupacional. A atividade profissional comporta ainda uma grande carga volitiva, na medida em que pode proporcionar um elevado status social, contribui para o sentido de autoeficácia do indivíduo e consequente aumento da autoestima, promove o relacionamento interpessoal e, estando aliado a interesses pessoais, é considerado uma fonte de satisfação.

Para o toxicodependente que tem uma atividade profissional, quando tem conhecimento da sua infecção pelo HIV, essa última parece ser a principal causa para o possível abandono do trabalho. Por outro lado, na função produtiva, apesar de o envolvimento e a responsabilidade poderem estar afetados, caso consiga manter o trabalho esse será um bom fator para avaliar positivamente a sua competência ocupacional (BRAVEMAN; KIELHOFNER, 2006). Essa última, pela sua natureza multidimensional, alia o desempenho, o significado e os contextos onde são realizadas ocupaçôes. Nesse sentido, não são só os locais de trabalho que têm importância, são também a casa deles ou os locais onde participam na educaçáo e no lazer. Todos influenciam a competência ocupacional ao permitirem o uso significativo do seu tempo e ao permtirem a assunção de papéis na vida. Veja-se a esse nível um detalhe da investigação, pelo qual os índices de competência ocupacional são inferiores nos indivíduos infectados por HIV: habitam quase todos em meio urbano, nas zonas de consumo ou próximo delas, afastando-se progressivamente de suas famílias de origem e dos outros contextos sociais (NEGREIROS, 2006). Isso faz com que diminuam os seus interesses e atividades significativas na área de ocupação lazer. Como esse tipo de atividade ensina novos métodos para lidar com o stress e com as experiências de sucesso sentidas, essas competências vão também diminuir. Assim diminuem as possibilidades para situações que promovem feedback realístico acerca de suas capacidades.

É de referir que parece existir um maior cuidado para a infecção pelo HIV nos comportamentos preventivos relacionados com o consumo injetado, mas náo existe a mesma prudência com outros tipos de comportamento de risco de infecção por HIV, como através de práticas sexuais (BASTOS, 2005; NEGREIROS et al., 2005). E a utilização do preservativo, de um modo correto e consistente, representa uma forma de prevenção eficiente (POMBO, 2003; DEREN et al., 2008). No nosso trabalho, são estatisticamente significativos os resultados referentes à frequência de utilização do preservativo e do seu uso na última relação sexual.

Num estudo efetuado por Friedman et al. (1999) com 1.240 toxicodependentes, a percentagem de toxicodependentes infectados por HIV que usa sempre o preservativo é de $46 \%$, sendo ligeiramente superior quando comparada com a de outros toxicodependentes. Ao comparar esses resultados com os da nossa investigação, essa diferença entre os grupos é ainda mais evidente, já que perto de três quartos dos toxicodependentes infectados por HIV relata usar sempre o preservativo, enquanto nos outros dois grupos apenas um quarto o faz. Efetivamente, o conhecimento da seropositividade para o HIV diminui os comportamentos sexuais de risco nos toxicodependentes (FRIEDMAN et al., 1999; DEREN et al., 2008). Contudo, o conhecimento do seroestatuto não anula os comportamentos sexuais de risco e alguns indivíduos vão continuar a manter esses comportamentos Também os dados do European Monitoring Centre for Drug and Drug Addiction (2001) corroboram nossos resultados, referindo que perto de dois terços dos toxicodependentes nunca usam preservativos com os parceiros habituais e perto de um terço nunca o usa com parceiros ocasionais. É de notar que 15,2\% dos toxicodependentes infetados por HIV, no nosso trabalho, não usaram preservativo em sua última relação sexual. Para esses indivíduos, o conhecimento da sua seropositividade em relação ao HIV não foi suficiente para adotarem comportamentos sexuais preventivos de forma consistente. Segundo Sparrow (2007), os comportamentos sexuais de risco podem não ser percebidos pelos toxicodependentes como 
uma autoameaça importante quando comparados com outros riscos sociais e de saúde com que se confrontam diariamente. É de sublinhar que a atividade sexual dessa população específica será responsável por aproximadamente metade de novas IST. Os consumidores de drogas têm tendência para usar menos os preservativos do que os iguais náo consumidores (SHARMA; AGGARWAL; DUBEY, 2002). Essas informaçôes, tal como os dados por nós obtidos, dão suporte à urgência de um novo alvo na prevenção da infecção por HIV nessa população: os toxicodependentes sem (qualquer) IST. Pois quando um toxicodependente é confrontado com a notícia da sua seropositividade ao HIV parece existir um comportamento comum quanto ao uso de precauçóes (preservativo) e redução dos riscos em todo o tipo de relaçóes sexuais.

Quando questionados sobre que outras atividades lhes dá satisfação para além do objeto de desejo (i.e., substância psicoativa que consomem), um grande número de toxicodependentes responde gratificação sexual. Devido à impulsividade e dificuldade de sentir tristeza desses indivíduos, a atividade sexual não é pensada ou programada, surge como substituto das sensações próprias das substâncias psicoativas de eleição e, como para tal, não observam os cuidados preventivos para com as DST (CORVINELLI, 2007). Esse imediatismo nas relaçóes sexuais faz ainda com que tenham vários parceiros sexuais ao longo do tempo e que tenham pouco cuidado consigo mesmos e com os outros (SHARMA; AGGARWAL; DUBEY, 2002). O número de parceiros sexuais é um dos fatores mais importantes em relação à possibilidade de uma DST, pois quanto maior o número de parceiros sexuais, em períodos curtos de tempo, maior é a probabilidade de infecção e transmissão de uma DST (KALICHMAN, 1998). Caso não esteja infectado com HIV, o toxicodependente, nessas situaçôes, coloca a responsabilidade da informaçâo serológica no outro e aguarda a respectiva solicitação dos comportamentos reventivos. Isso faz com que exista um clima de passividade generalizada, que propicia a propagaçáo do HIV. Assim, imerso em um ciclo em que tem muitas dificuldades para mudar as circunstâncias e realizar novas escolhas, o toxicodependente vai tornando a volição (i.e. predisposiçáo inata do homem para a ação) menos adaptativa. O valor mais alto encontrado para a volição foi no grupo A. Nesse sentido podemos afirmar que quanto maior é o valor da volição, melhor é a forma como o indivíduo percebe a eficiência, se satisfaz e diverte nas atividades que desenvolve nos diferentes papéis ocupacionais.
Para além da volição foram encontradas diferenças estatisticamente significativas na forma como percebem a competência ocupacional e a capacidade de desempenho. Para a dimensão competência ocupacional verifica-se que o grupo dos toxicodependentes infectados por HIV é o que percebe de forma mais diminuída a capacidade de desempenhar em equilíbrio e satisfaçáo os diferentes papéis ocupacionais. Por outro lado, é o grupo dos infectados por HIV o que mostra maior conhecimento em relação à doença e tem mais cuidado de proteção em relação à saúde, para si e para com os outros, quando do desempenho do papel sexual. Mas não é o desempenho muito satisfatório num só papel que faz com que o toxicodependente infectado com HIV perceba positivamente a competência ocupacional. O ser humano é essencialmente um ser ocupacional, que utiliza várias ocupaçôes de forma equilibrada como meio de interação com o mundo, criando e mantendo o sentido de pertença. E é assim que melhora a perceção da competência ocupacional e da sua saúde. Será através da descoberta, envolvimento e experimentação em diferentes ocupaçóes que o toxicodependente infectado com HIV logrará se regular emocionalmente, perceberá o seu potencial e criará qualidade de vida. Também Yallop (2000) refere que, após a infecção por HIV, o papel sexual é aquele com o qual gastam mais o tempo a realizar atividades associadas e o que passa a ganhar mais importância em detrimento de todos os outros. Mas para desempenhar o papel sexual de forma satisfatória e que não seja prejudicial à saúde, são necessárias as mesmas competências da área de ocupação produtividade (YALLOP, 2000). No entanto, apesar dos aspectos físicos, emocionais e comunicacionais serem similares para uma adequada performance no papel sexual e no papel produtivo, o grupo de toxicodependentes infectados por HIV apresenta maior funcionalidade no desempenho do papel sexual. Quanto ao papel produtivo, os dados estatisticamente significativos relativos à situação laboral e fonte de rendimento principal mostram que o grupo de toxicodependentes infectados por HIV apresenta déficits no seu desempenho. Nesse grupo de indivíduos, o comportamento ocupacional encontra-se em maior desequilíbrio. É devido a essa situação que os programas de ocupação, com orientaçôes precisas e intervenção diretiva, são fundamentais para a descoberta das capacidades pessoais, para a promoçáo da comunicação adequada com os outros e para a decisão acerca do futuro profissional (MAGALHÃES, 2000).

Christiansen (1999) enfatiza a correlação positiva, estatisticamente significativa, entre a saúde em geral 
e as competências nas áreas de ocupação. $\mathrm{O}$ mesmo autor refere nos seus estudos que o aparecimento de uma doença com implicações fisiológicas e psicossociais, como é o caso do HIV/SIDA, vai influenciar negativamente o indivíduo. Por um lado, influencia na satisfação e na importância atribuída às atividades realizadas no dia a dia. E, por outro, influencia no conhecimento e avaliação das capacidades reais dos diferentes papéis ocupacionais desempenhados.

Apesar de os seus resultados não serem estatisticamente significativos, sublinhe-se que em todos os grupos a satisfação ocupacional é muito baixa, pois a média é baixa, e os valores sáo muito discrepantes por haver um desvio padrão muito alto. Por conseguinte, isso significa que não há um padrão da satisfação ocupacional.

Pelos resultados obtidos podemos depreender que dos comportamentos sexuais estudados quatro são estatisticamente significativos quando comparamos as dimensóes e sub-escalas da OSA entre os grupos. Conclui-se que para a população toxicodependente, de acordo com o seu seroestatuto em relaçáo ao HIV, existe uma influência na forma como percebe a sua competência ocupacional em função de alguns comportamentos sexuais.

A população toxicodependente refere ter uma vida sexual ativa apresentando elevados índices de mudança de parceiros sexuais, o que potencia a transmissão do HIV (ROSENGARD; ANDERSON; STEIN, 2004). Repare-se que mais de metade teve/ tem encontros sexuais casuais com desconhecidos. Sublinham-se mais dois resultados que o atestam e que estão relacionados com a dimensão competência ocupacional: menos de um terço dos indivíduos referem zero como número de parceiros sexuais no último mês e em todos os grupos do estudo a competência ocupacional é percebida de forma superior se a resposta for um parceiro sexual no último mês. Uma a três vezes por semana é a frequência de atividade sexual mais escolhida em todos os grupos, mas é com a resposta todos ou quase todos os dias que os indivíduos dos diferentes grupos percebem ter uma competência ocupacional superior. É devido a serem sexualmente ativos e realizarem mudanças de parceiros sexuais que Loxley (2000) associou o aumento dos riscos de infecção a nível sexual em detrimento dos riscos na partilha de material para uso endovenoso.

Em relação à variável uso do preservativo na última relação sexual, a competência ocupacional é sentida de forma superior se os indivíduos usaram preservativo. Esse resultado parece indicar que os toxicodependentes, independentemente do seu seroestatuto em relação ao HIV, têm conhecimento de quais condutas terão influência no seu desempenho ocupacional. E, por conseguinte, a competência ocupacional não é influenciada pelo nível de conhecimento em relação ao HIV/SIDA (o conhecimento em relação ao HIV não é estatisticamente significativo quando comparamos a competência ocupacional entre os grupos).

Por outro lado, o grau de importância com que cada indivíduo classifica as mesmas áreas de funcionamento da competência ocupacional, a que corresponde a dimensão valores da OSA, apresenta resultado estatisticamente significativo no conhecimento em relação ao HIV. Por conseguinte, os resultados estatisticamente significativos nos comportamentos sexuais, quando comparamos os valores entre os grupos, são os que se relacionam com o uso do preservativo. A perceção da aprovaçáo social pela adoção de um determinado comportamento aumenta a probabilidade de a pessoa se envolver nesse comportamento. As normas sociais são um elemento influente na adoção do preservativo (BUUNK et al., 1998). O uso e frequência do preservativo parece, dessa forma, apelar à perscrutação da identidade ocupacional dos indivíduos, onde se incorporam os seus valores, sem a qual pode ser difícil compreender os significados associados ao uso de proteção sexual e propor formas de intervenção eficazes.

A inexistência de outras investigaçóes realizadas relativamente aos comportamentos sexuais e competência ocupacional em toxicodependentes levou-nos a sentir algumas dificuldades na discussão de alguns aspetos teóricos. Parece-nos que os toxicodependentes não são uma população homogênea de estudo, contudo a análise dos dados em cada investigação ajudará a identificar quais os fatores de risco específicos associados com a infecçâo por HIV e seus preditores. São duas possíveis limitaçôes deste estudo: o número de indivíduos da amostra poderia ser superior, de forma a validar alguns resultados que são apenas tendenciais; e o fato de basearmos as conclusões em autodescrições do comportamento, suscetíveis, por isso, de serem falseadas.

\section{Conclusão}

Existem diferenças entre os toxicodependentes de acordo com a presença ou ausência da infeção por HIV. Neste trabalho, essas diferenças evidenciaram-se, quer na adoção de comportamentos sexuais considerados de risco, quer na forma como percebem a competência ocupacional. Um toxicodependente infectado por HIV tem uma avaliação mais negativa da sua 
competência ocupacional quando comparado com outros toxicodependentes não infectados. Assume como diminuída a sua capacidade de desempenho em todos os seus papéis, nas diferentes áreas de ocupação. A exceção é o papel sexual. De forma um pouco paradoxal, são os toxicodependentes infectados com HIV os que adotam menos comportamentos sexuais de risco no desempenho desse papel. Como a competência ocupacional é a forma como sente que desempenha os seus diferentes papéis, no papel sexual ele o faz, e sabe que o faz, com vistas à sua proteção e à proteção da saúde dos seus parceiros sexuais. Também é diferente a competência ocupacional dos sujeitos com uma DST que pode ser tratada dos outros sujeitos com HIV, que ainda náo tem cura conhecida. Alguns dos dados aferidos no decorrer desse processo de investigação revelam ainda que, em relaçáo aos outros papéis ocupacionais, os toxicodependentes infectados por HIV consomem mais várias substâncias; apresentam maior percentagem do gênero feminino; vivem predominantemente em meio urbano; vivem em maior número em habitação degradada ou quarto alugado/pensão; vivem sozinhos, ou com amigos, longe da família de origem; têm uma situação laboral precária ou estáo desempregados; e a sua fonte de rendimento principal é o rendimento social de inserção/subsídios.

Como os diferentes componentes motores, cognitivos e de comunicaçáo são os mesmos quer para o papel sexual quer para todos os outros, os resultados da nossa investigação tornam plausível ser o conhecimento em relação à seropositividade ao HIV que faz a diferença em relaçáo à competência ocupacional percebida pelos toxicodependentes. Os toxicodependentes infectados por HIV têm, na sua maioria, um adequado desempenho ocupacional no seu papel sexual, pela diminuição dos comportamentos sexuais considerados de risco. Os requisitos, ao serem os mesmos para todos os papéis, também apresentam a possibilidade de se ter um adequado desempenho ocupacional nos outros papéis ocupacionais, mas percebem o seu seroestatuto como o declínio das suas funçóes ocupacionais. O papel sexual é a exceção da competência ocupacional, pois a maioria das atividades significativas que realizam estão centradas em serem pessoas infectadas por HIV.

Parece-nos que as intervençóes com vistas à informação das consequências do consumo endovenoso e partilha de material de injeçáo numa IST estáo apreendidas pelos toxicodependentes, pela diminuição dos comportamentos de risco a esse nível. Mas o mesmo náo se passa em relação aos comportamentos sexuais. Apesar da grande atividade sexual, e muitas vezes, com parceiros ocasionais, os toxicodependentes sem DST fazem pouco uso do preservativo. Desse modo se compreende a manutenção da percentagem de notificaçôes de HIV associadas à toxicodependência em Portugal. Os comportamentos sexuais de risco deveriam ser o novo foco das intervençóes junto a essa população específica, de forma a diminuir as novas infecções por HIV. O fator comum da transmissão e da prevenção do HIV é o comportamento. Contudo focar somente os aspectos relacionados com a sexualidade não nos parece suficiente. Por conseguinte, uma das informaçôes que podem ser veiculadas com vistas a diminuir novas infecções é a implicação que a infecção por HIV tem para o seu desempenho nos papéis das diferentes áreas de ocupação, nomeadamente, no que concerne à sua competência ocupacional.

Alguns dos resultados encontrados na presente investigação sugerem novos estudos a explorar em trabalhos futuros. Um aspecto a exigir uma investigação mais aprofundada relaciona-se a avaliação da competência ocupacional e estudo dos comportamentos sexuais de risco com outros tipos de dependência, nomeadamente, a população alcoólica, comparando-se com os resultados deste trabalho.

Acreditamos que a falta de cura para a infecção por HIV, no momento em que foi realizada esta investigação, afeta a realidade de hoje, mas será diferente daquela com que nos confrontaremos num amanhã. Acompanhar a evolução científica está condicionado à época, esta é efêmera e mantém-se válida e atual até ser substituída por novos construtos.

\section{Referências}

ADLER, N.; KEGELES, S.; GENEVRO, J. Risk taking and health. In: YATES, F. Risk-taking behavior. Chichester: John Wiley \& Sons, 1992. p. 231-256.

ANANDAN, N. et al. Impairments and perceived competence in persons living with HIV/AIDS. Work, Amsterdam, v. 27, n. 3, p. 255-266, 2006.

AZEVEDO, R.; BOTEGA, N.; GUIMARÃES, L. Crack users, sexual behavior and risk of HIV infection. Revista Brasileira de Psiquiatria, São Paulo, v. 29, n. 1, p. 26-30, 2007.

BARON, K. et al. The Occupational Self Assessment (OSA). Version 2.1. Chicago: The Model of Human Occupation Clearinghouse; Department of Occupational Therapy; University of Illinois, 2003.

BASTOS, J. VIH/SIDA e toxicodependência: mais vale prevenir. Toxicodependências, Lisboa, v. 11, n. 2, p. 73-81, 2005. 
BRAVEMAN, B.; KIELHOFNER, G. HIV/AIDS and employment: the continuing challenge. Work, Amsterdam, v. 27, n. 3, p. 205-207, 2006.

BUUNK, B. et al. Predictors of AIDS-preventive behavioral intentions among adult heterosexuals at risk for HIV infection: extending current models and measures. AIDS Education and Prevention, New York, v. 10, n. 2, p. 149-172, 1998.

CAREY, M. P.; MORRISON-BEEDY, D.; JOHNSON, B. T. The HIV-knowledge questionnaire: development and evaluation of a reliable, valid, and practical self-admnistered questionnaire. AIDS and Behavior, New York, v. 1, n. 1, p. 61-74, 1997. http://dx.doi.org/10.1023/A:1026218005943 CHRISTIANSEN, C. H. Defining lives, occupation as identity: an essay on competence, coherence and the creation of meaning. The American Journal of Occupational Therapy, Boston, v. 53, n. 6, p. 547-558, 1999. http:// dx.doi.org/10.5014/ajot.53.6.547

CORVINELLI, A. An emerging theory of boredom in recovery for adult substance users with HIV/AIDS attending an urban day treatment program. Occupational Therapy in Mental Health, New York, v. 23, n. 2, p. 27-50, 2007. http://dx.doi.org/10.1300/J004v23n02_02 COULDRICK, L. Sexual expression and occupational therapy. The British Journal of Occupational Therapy, London, v. 68, n. 7, p. 315-318, 2005.

CRUZ, J. F. A. Tradução e Adaptação do questionário The HIV-Knowledge Questionnaire de Carey, Morrison-Beddy e Johnson (1997). Braga: Universidade do Minho, 1998. (Manuscrito não publicado).

DEREN, S. et al. Sex risk behaviors of drug users: a dual site study of predictors over time. AIDS Education and Prevention, New York, v. 20, n. 3, p. 325-337, 2008. PMid:18673065. http://dx.doi.org/10.1521/ aeap.2008.20.4.325

EUROPEAN MONITORING CENTRE FOR DRUG AND DRUG ADDICTION - EMCDDA. Injecting drug use, risk behaviour and qualitative research in the time of Aids. Lisboa, 2001.

FISH, G.; RUDMAN, D. L. The potencial role of occupational therapy in acute care with clients with HIV/AIDS. Occupational Therapy International, London, v. 5, n. 1, p. 1-16, 1998. http://dx.doi.org/10.1002/oti.64 FORTIN, M. O processo de investigação. Loures: Lusociência, 2003.

FRIEDMAN, S. et al. Modulators of "Activated Motivation": event-specific condom use by drug injectors who have used condoms to prevent HIV/AIDS. AIDS and Behavior, New York, v. 3, n. 2, p. 85-98, 1999. http:// dx.doi.org/10.1023/A:1025427721485

GUERRA, M. Sida: implicaçôes psicológicas. Lisboa: Fim de Século, 1998.

JONES, M. K.; WEERAKOON, P.; PYNOR, R. A. Survey of occupational therapy students' attitudes towards sexual issues in clinical practice. Occupational Therapy
International, London, v. 12, n. 2, p. 95-106, 2005. http:// dx.doi.org/10.1002/oti.18

KALICHMAN, S. C. Preventing AIDS: a sourcebook for behavioural interventions. New Jersey: Lawrence Erlbaum Associates, 1998.

KIELHOFNER, G. Model of human occupation: theory and application. Baltimore: Lippincott Williams \& Wilkins, 2002. PMCid:PMC127022

KIELHOFNER, G. et al. Outcomes of a vocational program for persons with AIDS. The American Journal of Occupational Therapy, London, v. 58, n. 1, p. 64-72, 2004. http://dx.doi.org/10.5014/ajot.58.1.64

LAM, N. T. Drugs, sex and AIDS: sexual relationships among injecting drug users and their sexual partners in Vietnam. Culture, Health \& Sexuality, London, v. 10, p. 123-137, 2008. Supplement 1.

LOXLEY, W. Double risk: Young injectors and sexual relationships. Sexual and Relationship Therapy, London, v. 15, n. 3, p. 297-310, 2000. http://dx.doi. org/10.1080/14681990050109872

MAGAlHÂES, L. A doença é a dor de existir. Toxicodependências, Lisboa, v. 6, n. 1, p. 9-14, 2000.

NEGREIROS, J. Injecção de drogas, comportamento sexual e risco de VIH. Porto: Legis, 2006.

NEGREIROS, J.; MAGALHÃES, A. Contributos para a compreensão dos comportamentos de risco de transmissão do VIH em consumidores problemáticos de drogas. Toxicodependências, Lisboa, v. 11, n. 2, p. 3-22, 2005.

PEREIRA, A. Guia Prático de utilizaçâo do SPSS: análise de dados para Ciências Sociais e Psicologia. Lisboa: Sílabo, 2004.

POMBO, V. Sexualidade e Infecções Sexualmente Transmitidas (IST). In: Fonseca, L.; Soares, C.; Vaz, J. M. A sexologia: perspectiva multidisciplinar. Coimbra: Quarteto, 2003. p. 255-281. (v. 2).

ROSENGARD, C.; ANDERSON, B.; STEIN, M. Intravenous drug users' HIV-risk behaviors with primary/ other partners. The American Journal of Drug and Alcohol Abuse, New York, v. 30, n. 2, p. 225-236, 2004. PMCid:PMC1351211. http://dx.doi.org/10.1081/ ADA-120037375

ROSENGARD, C.; ANDERSON, B.; STEIN, M. Correlates of condom use and reasons for condom non-use among drug users. The American Journal of Drug and Alcohol Abuse, New York, v. 32, n. 4, p. 637-644, 2006. PMid:17127552. http://dx.doi. org/10.1080/00952990600919047

SALZ, F. HIV/AIDS and work: the implications for occupational therapy. Work, Amsterdam, v. 16, n. 3, p. 269-272, 2001.

SHARMA, A. K.; AGGARWAL, O. P.; DUBEY, K. K. Sexual behavior of drug-users: is it different? Preventive Medicine, New York, v. 34, n. 5, p. 512-515, 2002. PMid:11969351. http://dx.doi.org/10.1006/pmed.2002.1010 SOUSA, S. Avaliação ocupacional na psiquiatria: tradução, adaptação cultural e validação da versão portuguesa da 
OSA. 2006. 241 f. Tese (Mestrado em Psiquiatria e Saúde Mental)-Universidade do Porto, Porto, 2006.

SPARROW, P. Sex, drugs, and STIs: syphilis infection and hepatitis B vaccine compliance among illicit drug users in Houston. Houston: Faculty Health Science Center at Houston; University of Texas, 2007.

STOFFEL, V. C.; MOYERS, P. A. An evidence-based and occupational perspective of interventions for persons with substance-use disorders. The American Journal of
Occupational Therapy, Boston, v. 58, n. 5, p. 570-586, 2004. http://dx.doi.org/10.5014/ajot.58.5.570

YALLOP, S. Identity issues for people living with HIV/ AIDS: humans with potential or sexual victims? British Journal of Occupational Therapy, London, v. 63, n. 9, p. 419-424, 2000.

ZHAO, M. et al. HIV sexual risk behaviours among injection drug users in Shanghai. Drug and Alcohol Dependence, Lausanne, v. 82, p. 43-47, 2006. Supplement 1.

\section{Notas}

${ }^{1}$ Material parte de pesquisa em que todos os procedimentos éticos vigentes foram cumpridos.

${ }^{2}$ Informa-se que alguns dos resultados já foram apresentados no 7o Congresso Nacional de Terapia Ocupacional realizado nos dias 26 e 27 de outubro de 2012, ocorrido no Instituto de Investigação e Formação Avançada em Ciências e Tecnologias da Saúde de Penafiel (Portugal), organizado pela Associaçáo Portuguesa de Terapeutas Ocupacionais. 